\title{
Polarons in semiconductor quantum-dots and their role in the quantum kinetics of carrier relaxation
}

\author{
J. Seebeck, ${ }^{1}$ T.R. Nielsen, ${ }^{1}$ P. Gartner, ${ }^{1,2}$ and F. Jahnke ${ }^{1}$ \\ ${ }^{1}$ Institute for Theoretical Physics, University of Bremen, 28334 Bremen, Germany \\ ${ }^{2}$ National Institute for Materials Physics, POB MG-7, Bucharest-Magurele, Romania
}

(Dated: September 7, 2018)

\begin{abstract}
While time-dependent perturbation theory shows inefficient carrier-phonon scattering in semiconductor quantum dots, we demonstrate that a quantum kinetic description of carrier-phonon interaction predicts fast carrier capture and relaxation. The considered processes do not fulfill energy conservation in terms of free-carrier energies because polar coupling of localized quantum-dot states strongly modifies this picture.
\end{abstract}

PACS numbers: 73.21.La,78.67.Hc

\section{INTRODUCTION}

Applications of semiconductor quantum dots (QDs) in optoelectronic devices rely on fast carrier scattering processes towards and between the discrete confined levels. These carrier transitions determine the dynamics of QD luminescence [1] or the operation of QD lasers [2, 3]. For low carrier densities, where Coulomb scattering can be neglected, carrier-phonon interaction provides the dominant scattering channel. In QDs only phonons with small momenta can efficiently couple to the confined carriers [4]. Then interaction with LA phonons does not contribute for large transition energies and only quasimonochromatic LO phonons need to be considered.

The simplest theoretical approach to electronic scattering processes is based on time-dependent perturbation theory. Fermi's golden rule for carrier transitions due to phonon emission or absorption contains a deltafunction for strict energy conservation in terms of freecarrier energies of initial and final states and the phonon energy. When transition energies of localized QD states do not match the LO-phonon energy, efficient scattering is inhibited (leading to the prediction of a phonon bottleneck) and only higher-order processes, like a combination of LO and LA phonons [5, [6], weakly contribute. Attempts to broaden the delta-function "by hand" immediately change the results $[\underline{6}$, which underlines that this point should be addressed microscopically. The phonon bottleneck effect is still a debated topic, with experimental evidence both for [7, 8, 9] and against it [10, 11, 12].

As in any coupled system, carrier-phonon interaction renormalizes both electronic and vibrational states. However, in bulk semiconductors or quantum wells with weak polar coupling, the net effect can be described by renormalized effective carrier masses, a small polaron shift of the band-edge, and lattice distortions only modify the background dielectric constant for the Coulomb interaction of carriers. The broadening of the transition energies due to carrier-phonon interaction remains weak.

For carriers in QDs, the discrete nature of localized electronic states changes the role of polaronic effects [4, 13, 14]. Restricting the analysis to a single QD state coupled to phonons, polaron effects can be obtained from an exact diagonalization of the Hamiltonian [15]. While an extension to several discrete levels has been presented [16], the influence of the energetically nearby continuum of wetting-layer (WL) states, typical for self-assembled QDs, has not been included. Furthermore, only quasiparticle properties have been discussed which provide no direct information about the scattering efficiency for various processes. Calculations of carrier transition rates based on the polaron picture are missing.

We use a quantum kinetic treatment for carrier-phonon interaction in the polaron picture. As a first step, quasiparticle renormalizations due to the polar interaction for both QD and WL carriers are determined. For the QD states, the hybridization of one state with strong satellites of another state leads to a rich multi-peak structure. The WL states exhibit weak LO-phonon satellites. Coupling to the WL states provides a broadening mechanism for the QD states.

Based on the spectral properties of QD and WL polarons, quantum kinetic equations for the capture process (carrier transitions from the WL into the QD) and relaxation processes (transitions between QD states) are solved. For situations where, in terms of free-carrier energies, energy conserving scattering processes are not possible, the quantum-kinetic treatment provides efficient scattering rates. Even for the InGaAs material system with weak polar coupling, sub-picosecond scattering times are obtained.

\section{QUANTUM DOT POLARONS}

The single-particle properties of carriers under the influence of lattice distortions are determined by the retarded Green's function (GF), $G_{\alpha}^{r}$, which obeys the Dyson equation

$$
\begin{aligned}
& {\left[i \hbar \frac{\partial}{\partial t_{1}}-\varepsilon_{\alpha}\right] G_{\alpha}^{r}\left(t_{1}, t_{2}\right) }=\delta\left(t_{1}-t_{2}\right) \\
&+\int d t_{3} \quad \Sigma_{\alpha}^{r}\left(t_{1}, t_{3}\right) G_{\alpha}^{r}\left(t_{3}, t_{2}\right)
\end{aligned}
$$


Here $\alpha$ is an arbitrary (QD or WL) electronic state with free-carrier energy $\varepsilon_{\alpha}$. In the polaron theory one usually considers all possible virtual transitions from this state due to emission or absorption of phonons. This corresponds to a self-energy $\Sigma_{\alpha}^{r}$ for the carrier-phonon interaction where the population of the involved carrier states is neglected (electron vacuum). The corresponding retarded self-energy in random-phase approximation (RPA) is given by 17.

$$
\Sigma_{\alpha}^{r}\left(t_{1}, t_{2}\right)=i \hbar \sum_{\beta} G_{\beta}^{r}\left(t_{1}, t_{2}\right) D_{\beta \alpha}^{<}\left(t_{2}-t_{1}\right) .
$$

Assuming that the phonon system is in thermal equilibrium, the phonon propagator (combined with the interaction matrix elements) is given by

$$
\begin{aligned}
i \hbar D_{\beta \alpha}^{<}(\tau) & =\sum_{\boldsymbol{q}}\left|M_{\beta \alpha}(\boldsymbol{q})\right|^{2} \\
& \times\left[n_{L O} e^{-i \omega_{L O} \tau}+\left(1+n_{L O}\right) e^{i \omega_{L O} \tau}\right]
\end{aligned}
$$

where monochromatic LO-phonons with the frequency $\omega_{L O}$ are considered. The corresponding phonon population is given by $n_{L O}=1 /\left(e^{\hbar \omega_{L O} / k T}-1\right)$ and the Fröhlich interaction matrix element

$$
M_{\beta \alpha}(\boldsymbol{q})=\frac{M_{L O}}{q}\left\langle\beta\left|e^{i \boldsymbol{q} \boldsymbol{r}}\right| \alpha\right\rangle
$$

contains the overlap between the electronic states and the phonon mode. For localized electronic states this acts as a form factor. The prefactor $M_{L O}^{2}=4 \pi \alpha \frac{\hbar}{\sqrt{2 m}}\left(\hbar \omega_{L O}\right)^{3 / 2}$ includes the polar coupling strength $\alpha$ and the reduced mass $m$. As a result of the above assumptions, the retarded GF itself depends only on the difference of time arguments and its Fourier transform can be directly related to the quasi-particle properties.

Due to energy separation between the discrete QD states and the WL continuum, polaronic effects in QDs are often computed by neglecting the presence of the WL 4, 13, 14, 16]. For a single discrete level this amounts to the exactly solvable independent Boson model 15] and for several discrete levels it was shown to be nearly exactly solvable [16]. In both cases, even for non-zero temperatures, the spectral function contains a series of sharp delta-like peaks. In real QDs, however, the interaction with the WL continuum (which might require multi-phonon processes) leads to a broadening of these peaks. The RPA accounts for this broadening effect while it retains a hybridization effect (see below) characteristic for the full solution. Therefore the RPA is expected to provide an adequate description in the presence of the continuum [19]. An additional source of broadening is the finite LO-phonon lifetime due to anharmonic interaction between phonons.

For the numerical results presented in this paper we consider an InGaAs QD-WL system with weak polar coupling $\alpha=0.06$. The effective-mass approximation is as- sumed to be valid with $m_{e}=0.067 m_{0}$ for the conduction band. For flat lens-shaped QDs the in-plane wavefunctions of an isotropic two-dimensional harmonic potential are used while for the (strong) confinement in the direction perpendicular to the WL a finite-height potential barrier is considered (see 18] for parameters and further details). To account for a finite height of the QD confinement potential, the calculations only include the (double degenerate) ground state and the (four-fold degenerate) first excited state, in the following called $\mathrm{s}$ and p-shell, respectively with s-p spacing and p-WL separation of $40 \mathrm{meV}$. For the description of the WL states we use the following steps: (i) the WL states in the absence of QDs are described by plane waves for the in-plane part, multiplied by the state corresponding to the finite-height barrier confinement for the perpendicular direction. (ii) to describe the WL states in the presence of the QDs, the orthogonalized plane wave scheme, described in Appendix A of Ref. [18], is used to construct WL states orthogonal to the QD states. Calculations are done for a density of QDs on the WL $n_{d o t}=10^{10} \mathrm{~cm}^{-2}$. Details on the calculation of the interaction matrix elements in Eq. (4) with these wave functions for various combinations of QD and WL states can be found in Appendix B of Ref. [18]. Convergent results are obtained with 128 points for the in-plane momentum radial integrals and 50 points for the remaining angular integrations entering the interaction matrix elements.

The Fourier transform of the spectral function, -2 Im $G_{\alpha}^{r}(\omega)$, is shown in Fig. 10 for the $k=0 \mathrm{WL}$ state and for the QD p- and s-shell (from top to bottom). In the absence of polar coupling to lattice distortions, the spectral functions are delta functions at the free-particle energies indicated by the vertical lines. The dotted line in Fig. 1(a) is the result for the $k=0 \mathrm{WL}$ state without coupling to the QD states, indicating that their influence on the WL polarons is weak. The WL spectral function is broadened, the central peak exhibits a small polaron shift, and multiple sidebands due to LO-phonon emission (absorption) appear to the right (left). The polaron broadening is a result of the irreversible decay in the continuous WL density of states.

The LO-phonon sidebands of the localized QD states in Fig. [1 (b) and (c) are more pronounced and the hybridization of peaks from one shell with the energetically close sidebands of the other shell can be observed. This effect stems from the discrete nature of the localized states and requires that the coupling strength, which is modified by the form factors in Eq. (4), exceeds the polaron damping. If only coupling matrix elements diagonal in the state index would be considered, a series of sidebands of a state with discrete energy, spaced by the LO-phonon energy, would be obtained. Off-diagonal coupling elements alone would lead to a hybridization of discrete levels as, e.g., in quantum optics where instead of the phonon-field a monochromatic light field coupled to a two-level system is considered [4]. When the level splitting equals the LO-phonon energy, in the limit of weak damping the 


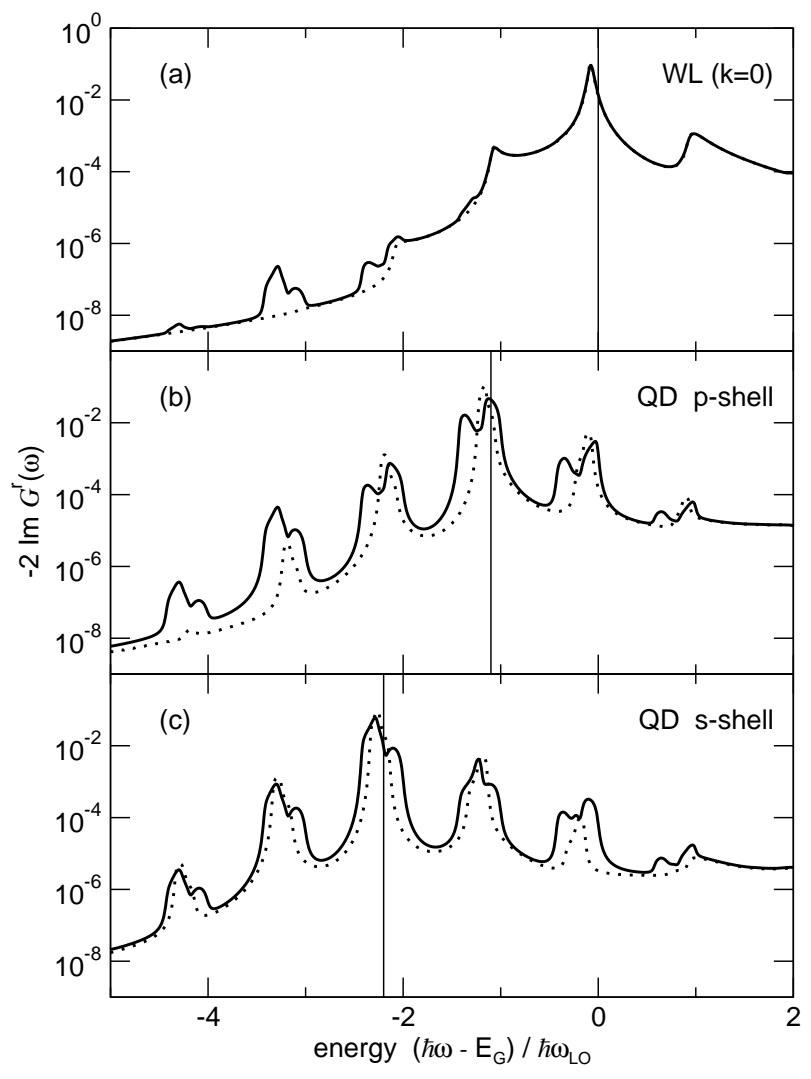

FIG. 1: (a) Spectral function of electrons for the lowest WL state at $k=0$ under the influence of polar coupling in the combined QD-WL system (solid line) and without coupling to the QD states (dotted line). For electrons in the QD pshell (b) and s-shell (c) full coupling between all states (solid line) is compared to the case without coupling to other QD states (dotted line). Vertical lines show the corresponding free-carrier energies. Energies are given relative to the continuum edge $E_{G}$ in units of the phonon energy $\hbar \omega_{L O}$. The temperature is $300 \mathrm{~K}$.

splitting of each line is determined by the carrier-phonon coupling strength. Due to the interplay of diagonal and off-diagonal interaction matrix elements, the QD spectral functions in Fig. 1(b) and (c) show a series of satellites, each of them reflecting the hybridization. The asymmetry stems from the difference between level spacing ( 40 $\mathrm{meV}$ ) and LO-phonon energy $(36 \mathrm{meV})$. The broadening of peaks stems mainly from the coupling to the $\mathrm{WL}$ states. A finite LO-phonon lifetime of 5 ps due to anharmonic interaction between phonons has been included in the calculations.

\section{CARRIER KINETICS OF RELAXATION AND CAPTURE PROCESSES}

In this section we study consequences of the renormalized quasi-particle properties on the scattering processes. Fermi's golden rule, which has been frequently used in the past, describes only transition rates from fully populated initial into empty final states. Proper balancing between in- and out-scattering events, weighted with the population $f$ of the initial states and the blocking $1-f$ of the final states, leads to the kinetic equation

$$
\begin{aligned}
& \frac{\partial}{\partial t} f_{\alpha}=\frac{2 \pi}{\hbar} \sum_{\beta, \boldsymbol{q}}\left|M_{\beta \alpha}(\boldsymbol{q})\right|^{2} \\
& \times\left\{( 1 - f _ { \alpha } ) f _ { \beta } \left[\left(1+n_{L O}\right) \delta\left(\varepsilon_{\alpha}-\varepsilon_{\beta}+\hbar \omega_{L O}\right)\right.\right. \\
&\left.+n_{L O} \delta\left(\varepsilon_{\alpha}-\varepsilon_{\beta}-\hbar \omega_{L O}\right)\right] \\
&-f_{\alpha}\left(1-f_{\beta}\right)\left[n_{L O} \delta\left(\varepsilon_{\alpha}-\varepsilon_{\beta}+\hbar \omega_{L O}\right)\right. \\
&\left.\left.+\left(1+n_{L O}\right) \delta\left(\varepsilon_{\alpha}-\varepsilon_{\beta}-\hbar \omega_{L O}\right)\right]\right\} .
\end{aligned}
$$

Quasiparticle and Markov approximation can also be applied to renormalized polaronic states which results in a rate-equation description for the population of these states [20].

A quantum-kinetic approach extends Eq. (5) in the sense that the delta-functions with free-carrier energies are replaced by time-integrals over polaronic retarded GFs. Furthermore, the population factors are no longer instantaneous but explicitly depend on the time evolution. This is the time-domain picture for the inclusion of renormalized quasi-particle properties (beyond a quasi-particle approximation and beyond Markov approximation). Using the generalized Kadanoff-Baym ansatz (GKBA) [21], the quantum-kinetic equation has the form

$$
\begin{aligned}
\frac{\partial}{\partial t_{1}} f_{\alpha}\left(t_{1}\right) & =2 \operatorname{Re} \sum_{\beta} \int_{-\infty}^{t_{1}} d t_{2} G_{\beta}^{r}\left(t_{1}-t_{2}\right)\left[G_{\alpha}^{r}\left(t_{1}-t_{2}\right)\right]^{*} \\
\times & \left\{\left[1-f_{\alpha}\left(t_{2}\right)\right] f_{\beta}\left(t_{2}\right) i \hbar D_{\alpha \beta}^{>}\left(t_{2}-t_{1}\right)\right. \\
& \left.-f_{\alpha}\left(t_{2}\right)\left[1-f_{\beta}\left(t_{2}\right)\right] i \hbar D_{\alpha \beta}^{<}\left(t_{2}-t_{1}\right)\right\} . \quad(6)
\end{aligned}
$$

The phonon propagator $D^{>}$follows from Eq. (3) by replacing $\tau \rightarrow-\tau$. A Markov approximation in the renormalized quasi-particle picture corresponds to the assumption of a slow time-dependence of the population $f_{\alpha}\left(t_{2}\right)$ in comparison to the retarded GFs such that the population can be taken at the external time $t_{1}$. The Boltzmann scattering integral of Eq. (5) follows if one additionally neglects quasi-particle renormalizations and uses free-carrier retarded GFs 22].

To demonstrate the influence of quantum-kinetic effects due to QD-polarons, we first study the relaxation of carriers from p-shell to s-shell for the above discussed situation where the level spacing does not match the LOphonon energy such that both, Fermi's golden rule and the kinetic equation (5) predict the absence of transitions. A direct time-domain calculation of the polaron GFs from Eq. (1)-(4) together with Eq. (6) is used. We assume an initial population $f_{s}\left(t_{0}\right)=0, f_{p}\left(t_{0}\right)=0.3$ and start the calculation at time $t_{0}$. While this example addresses the relaxation process itself, more advanced calculations would also include the carrier generation via optical excitation or carrier capture discussed below. Then 


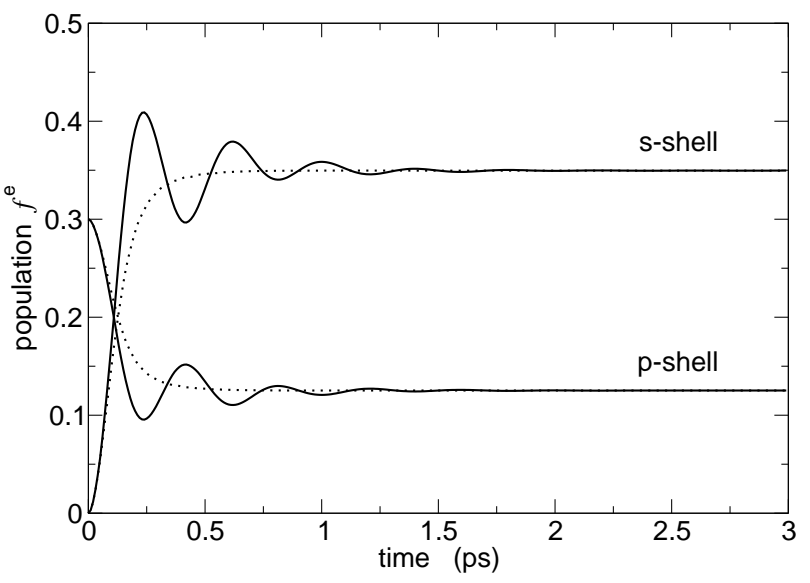

FIG. 2: Temporal evolution of the QD population due to carrier-phonon scattering between p-shell and s-shell for an energy spacing larger than the LO-phonon energy. The solid lines correspond to a quantum-kinetic calculation whereas for the dotted line the Markov approximation is used together with polaronic spectral functions.

ambiguities due to initial conditions can be avoided since the population vanishes prior to the pump process which naturally provides the lower limit of the time integral in Eq. (6). In practice, we find that within the GKBA results weakly depend on the details of the initial conditions.

The evaluation of the quantum-kinetic theory (solid lines in Fig. (2) yields a fast population increase of the initially empty QD s-shell accompanied by oscillations which reflect in the time-domain the hybridization of coupled carrier and phonon states. The analogy to Rabi oscillations has been pointed out in Ref. [4]. If one uses the Markov approximation together with polaronic retarded GFs in Eq. (6), such that quasi-particle renormalizations are still included, these transient oscillations disappear. In both cases the same steady-state solution is obtained which corresponds to a thermal population at the renormalized energies. The equilibrium solution can be obtained from the polaron spectral function using the Kubo-Martin-Schwinger (KMS) relation, $f_{\alpha}=-\int \frac{d \hbar \omega}{\pi} f(\omega) \operatorname{Im} G_{\alpha}^{r}(\omega)$ where $f(\omega)$ is a Fermi function with the lattice temperature. Note that particle number conservation is obeyed in Fig. 2 since the degeneracy of the p-shell is twice that of the s-shell.

Another important process is the capture of carriers from the delocalized WL states into the localized QD states. For the used QD parameters, where the spacing between the p-shell and the lowest WL state $(40 \mathrm{meV})$ exceeds the LO-Phonon energy, again Fermi's golden rule and Eq. (5) predict the absence of electronic transitions. For the numerical solution of Eq. (6) we use now as initial condition empty QD states and a thermal population of carriers in the polaronic WL states (obtained from the KMS relation) corresponding to a carrier density $10^{11}$ $\mathrm{cm}^{-2}$ and temperature $300 \mathrm{~K}$ 23. The dashed-dotted and dotted lines in Fig. 3 show the increase of the p-

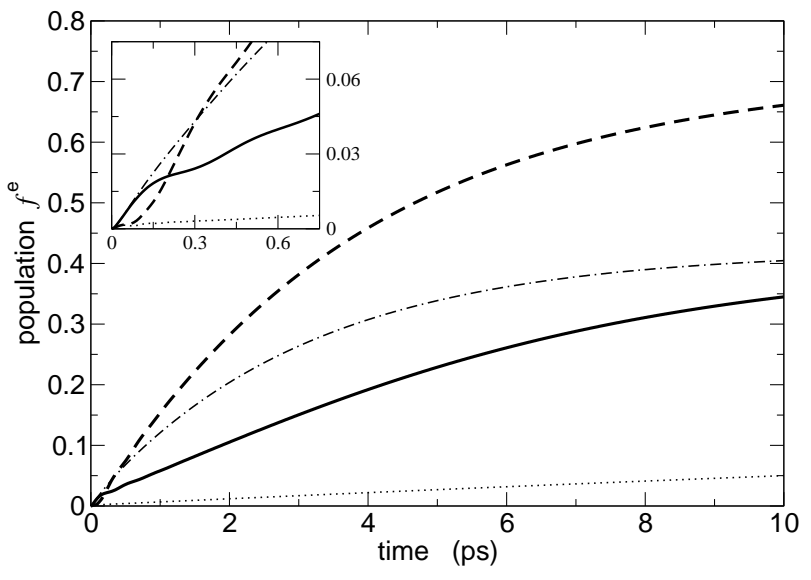

FIG. 3: Time-evolution of the QD p-shell (solid line) and sshell (dashed line) electron population due to carrier capture from the WL including the effect of carrier relaxation between QD shells. If only direct capture processes are considered, the dashed-dotted and dotted lines are obtained for p-shell and s-shell, respectively.

and s-shell population, respectively, when only capture processes are considered (scattering from a WL-polaron to a QD-polaron state due to emission of LO-phonons). Also in this situation the quantum-kinetic theory predicts a fast population of the initially empty p-shell. Albeit the large detuning (exceeding two LO-phonon energies) the direct capture to the s-shell is still possible but considerably slower. When both, direct capture of carriers as well as relaxation of carriers between the QD states are included in the calculation, the solid (dashed) line is obtained for the p-shell (s-shell) population. While faster capture to the p-shell states leads at early times to a p-shell population exceeding the s-shell populations (see inset of Fig. 3), the subsequent relaxation efficiently populates the s-states. Since the WL states form a quasicontinuum, beating at early times is strongly suppressed.

With the results in Figs. [1.3] we have demonstrated the ultrafast (subpicosecond) carrier relaxation and fast (picosecond) carrier capture for a material with weak polar coupling and 10\% detuning between the transition energies and the LO-phonon energy. This detuning is on the one hand sufficiently large for the alternative LO+LA mechanism proposed by Inoshita and Sakaki 5 to fail and on the other hand small enough to illustrate the hybridization of one state with sidebands of the other states. We find that the fast scattering is not related to the near resonance condition and in fact relatively insensitive to the detuning between transition energies and LO-phonon energy. The spectral functions of the coupled QD-WL system for various detunings, ranging from resonance to

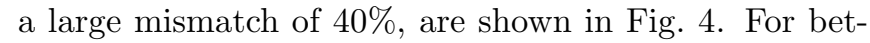
ter visibility only the curves for the s- and p-shells are displayed, while the WL-states are also included in the calculations. As seen in Fig. 1 the spectral function for the WL states is only weakly influenced by the coupling to the QDs. In all three cases of Fig. 4 there is sub- 


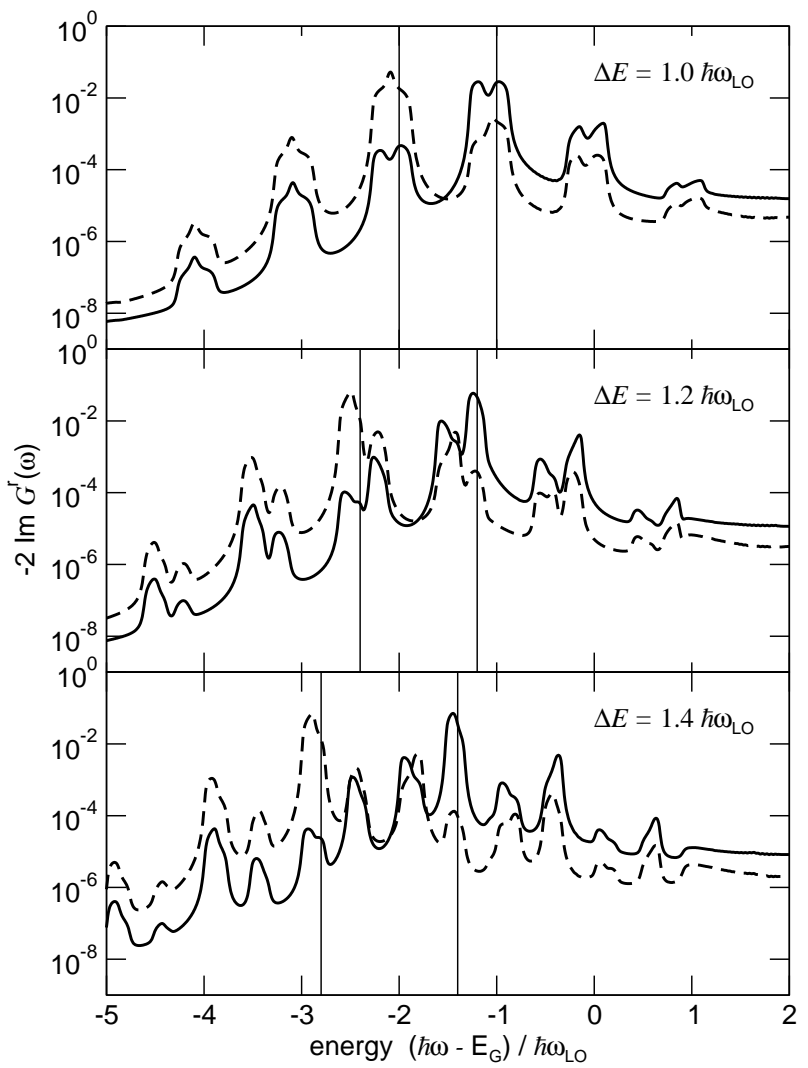

FIG. 4: (a) Spectral function of electrons in the coupled QDWL system for the p-shell (solid line) and s-shell (dashed line) using various energy spacings $\Delta E$ between s-shell, p-shell and WL $(k=0)$ in units of the LO-phonon energy $\hbar \omega_{L O}$. Vertical lines indicate the positions of the unrenormalized QD states, $E_{G}$ is the continuum edge of the WL states. The temperature is $300 \mathrm{~K}$.

stantial overlap between the s-shell and p-shell density of states which points to efficient transition processes. This overlap is due to the multi-peak-structure which contains the series of phonon sidebands spaced by the LO-phonon energy and their hybridization. From top to bottom in Fig. [4 the peak splitting increases with detuning.

The corresponding results for the carrier relaxation, as in Fig. 2 but for different detunings $\Delta E$, are shown in Fig. 5 The fast carrier relaxation towards an equilibrium situation is retained in all three cases. The main difference is in the oscillation period, which is reduced for larger detuning due to the increased splitting in Fig. 4.

A stronger influence of the detuning between transition energies and the LO-phonon energy is found for the capture of carriers from the WL into the QD states. As can be seen in Fig. [6 from the resonance situation to a detuning of $40 \%$ the capture efficiency is reduced by about one order of magnitude. Nevertheless, a significant occupancy can be reached within several ten picoseconds. The reduced capture efficiency is related to a reduced overlap between the WL and QD spectral functions for increasing detuning (which is mainly because the WL states are

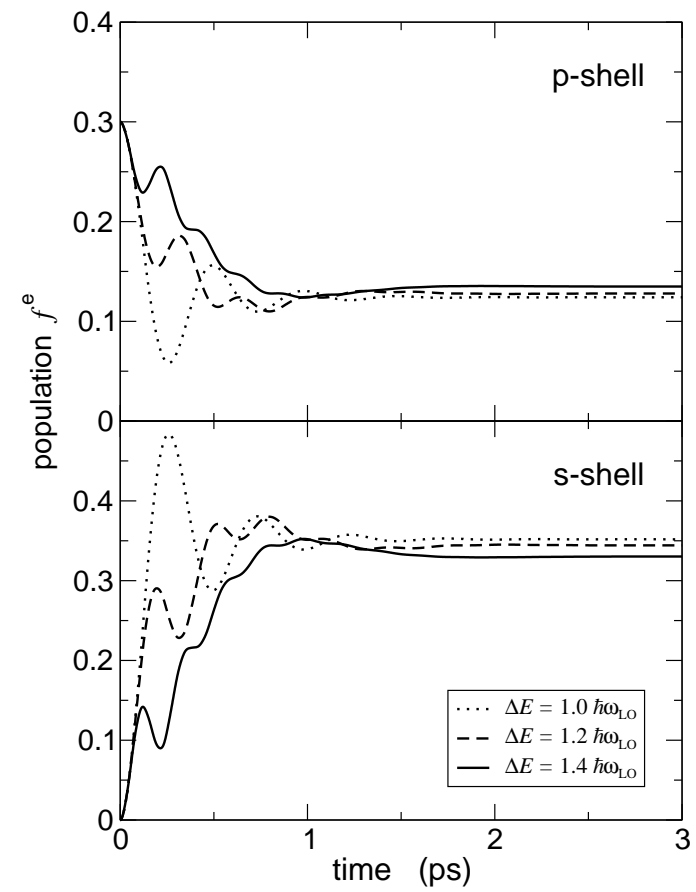

FIG. 5: Temporal evolution of the QD population due to carrier-phonon scattering between p-shell (initially populated) and s-shell (initially empty) for different energy spacings $\Delta E$ corresponding to Fig. 4

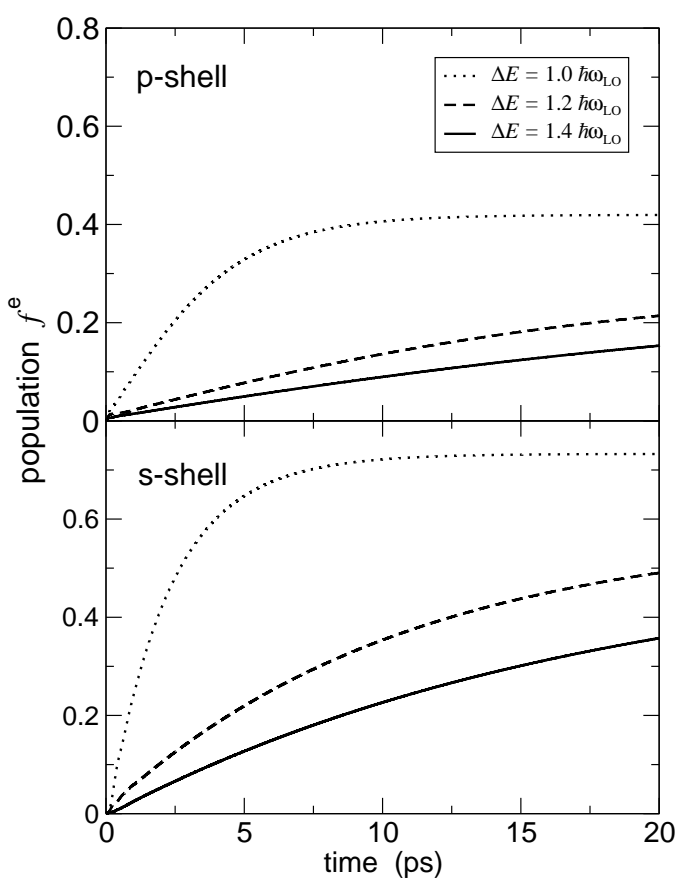

FIG. 6: Time-evolution of the QD p-shell (top) and s-shell (bottom) electron population due to carrier capture from the WL including the effect of carrier relaxation between QD shells for different energy spacings $\Delta E$ corresponding to Fig. 4

weakly influenced by the QD states). In comparison to 
this, the strong interaction between s- and p-states maintains a strong overlap between their spectral functions. As a consequence the relaxation time is less sensitive to the detuning.

In summary, the quantum-kinetic treatment of carrierphonon interaction explains the absence of a phonon bottleneck in terms of scattering between renormalized quasi-particle states. A quasi-equilibrium situation is reached on a ps-timescale at elevated temperatures even in materials with weak polar coupling.

\section{Acknowledgments}

This work was supported by the Deutsche Forschungsgemeinschaft and with a grant for CPU time at the NIC, Forschungszentrum Jülich.
[1] D. Morris, N. Perret, and S. Fafard, Appl. Phys. Lett. 75, 3593 (1999).

[2] P. Bhattacharya, K.K. Kamath, J. Singh, D. Klotzkin, J. Phillips, H.-T. Jiang, N. Chervela, T.B. Norris, T. Sosnowski, J. Laskar, M.R. Murty, IEEE Transactions on Electron Devices 46, 871 (1999).

[3] G. Park, O.B. Shchekin, D.L. Huffaker, and D.G. Deppe, Electron. Lett. 36, 1283 (2000).

[4] T. Inoshita and H. Sakaki, Phys. Rev. B 56, 4355 (1997).

[5] T. Inoshita and H. Sakaki, Phys. Rev. B 46, 7260 (1992).

[6] H. Jiang and J. Singh, IEEE J. Quantum Electron. 34, 1188 (1998).

[7] J. Urayama, T. B. Norris, J. Singh, and P. Bhattacharya, Phys. Rev. Lett. 86, 4930 (2001).

[8] A.W.E. Minnaert, A.Yu. Silov, W. van der Vleuten, J.E.M. Haverkort, and J.H. Wolter, Phys. Rev. B 63, 075303 (2001).

[9] S. Xu, A.A. Mikhailovsky, J.A. Hollingsworth, and V.I. Klimov, Phys. Rev. B 65, 045319 (2002).

[10] E. Tsitsishvili, R. v. Baltz, and H. Kalt, Phys. Rev. B 66, 161405 (2002)

[11] E. Peronne, F. Fossard, F.H. Julien, J. Brault, M. Gendry, B. Salem, G. Bremond, and A. Alexandrou, Phys. Rev. B 67, 205329 (2003).

[12] F. Quochi, M. Dinu, L.N. Pfeiffer, K.W. West, C. Kerbage, R.S. Windeler, and B.J. Eggleton, Phys. Rev. B 67, 235323 (2003).

[13] K. Kral and Z. Khas, Phys. Rev. B 57, 2061 (1998).

[14] O. Verzelen, R. Ferreira, G. Bastard, T. Inoshita, H. Sakaki, phys. stat. sol. (a) 190, 213 (2002).

[15] G.D. Mahan, Many-Particle Physics (Plenum, New York, 1990).
[16] T. Stauber, R. Zimmermann, and H. Castella, Phys. Rev. B 62, 7336 (2000).

[17] In general, Green's functions and self-energies depend on two quantum numbers $\alpha, \beta$. For the situation described in this paper, off-diagonal contributions can be neglected, which has been verified numerically; see also [4, 13] .

[18] T.R. Nielsen, P. Gartner, and F. Jahnke, Phys. Rev. B 69, 235314 (2004).

[19] Comparison with an alternative method supports this statement: the first term of a cumulant expansion, which is known to be exact for the independent Boson model 15], agrees well with the RPA for quasi-continuous electronic states.

[20] O. Verzelen, R. Ferreira, and G. Bastard, Phys. Rev. B 62, 4809 (2000).

[21] for a review see: H. Haug and P. Jauho, Quantum Kinetics in Transport 85 Optics of Semiconductors, SpringerVerlag, Berlin, 1996.

[22] The quantum-kinetic equation provides energyconserving scattering processes only in the long-time limit. The corresponding spectral function in Fig. 1] is obtained from a complete Fourier transform whereas on a short timescale the peaks shown there are only partially emerged (since only a finite-time contribution of the retarded GF enters in the quantum-kinetic equation at a given time.)

[23] For small carrier densities, we use the polaron GF for the electron vacuum since it is weakly influenced by population effects, see P. Gartner, L. Bányai, and H. Haug, Phys. Rev. B 60, 14234 (1999). 